\title{
Recomendações de Acessibilidade para Surdos dos Tipos de Questões Usadas na Avaliação Baseada em Computador em Ambientes Virtuais de Aprendizagem
}

\author{
Maíra Codo Canal ${ }^{1}$, Laura Sánchez García ${ }^{1}$ \\ ${ }^{1}$ Departamento de Informática - Universidade Federal do Paraná (UFPR) \\ Caixa Postal 19.081 - 81.531-980 - Curitiba - PR - Brasil \\ mairapdnegmail.com, lauraeinf.ufpr.br
}

\begin{abstract}
The types of questions available for Computer-Based Assessment (CBA) in Virtual Learning Environments (VLEs) still present accessibility issues in the context of deaf students. Due to these problems, the interaction of deaf students can be impaired and, consequently, deaf students can be prevented of using types of questions in VLEs as others students do. This work presents a set of accessibility recommendations for the deaf of the types of questions used in CBA in VLEs. The set of recommendations proposed by this research was evaluated by IT experts and by deaf teachers. The evaluation results determined positive feedback both regarding the usefulness of the recommendations like their accuracy.
\end{abstract}

Resumo. Os tipos de questões que são usadas na Avaliação Baseada em Computador (CBA, do inglês Computer-Based Assessment) em Ambientes Virtuais de Aprendizagem (AVAs) possuem problemas de acessibilidade no contexto de estudantes surdos. Devido a esses problemas, a interação dos estudantes surdos com os tipos de questões fica comprometida e, dessa maneira, os estudantes surdos podem ficar impedidos de utilizar os tipos de questões em AVAs como os demais estudantes. Este trabalho apresenta um conjunto de recomendações de acessibilidade para surdos dos tipos de questões usadas na CBA em AVAs. O conjunto de recomendações deste trabalho foi avaliado por especialistas em Tecnologia da Informação (TI) e por professoras surdas. Os resultados dessa avaliação determinaram posicionamentos positivos tanto sobre a utilidade do uso das recomendações, como em relação à sua precisão, clareza e completude.

\section{Introdução}

A avaliação é reconhecida como um elemento-chave na aprendizagem, uma vez que tem potencial de capacitar, direcionar, motivar os alunos e fornecer critérios de sucesso para medir o progresso da aprendizagem. A informatização do processo de avaliação pode tornar-se um avanço educacional, pois automatiza e facilita os procedimentos lentos e tediosos envolvidos no planejamento, na entrega e na análise da avaliação [Sim et al. 2004]. CBA pode ser composta de diferentes tipos de questões, como: verdadeiro/falso, múltipla escolha, dissertação, entre outros.

É necessário assegurar que CBA possa ser utilizada por todos, incluindo as pessoas com deficiência. Estudos relatam problemas de acessibilidade em tipos de 
questões utilizadas na CBA. Luephattanasuk et al. (2011) afirmam que recomendações mais específicas são necessárias para identificar problemas de acessibilidade para que pessoas com deficiência possam interagir com os tipos de questões utilizadas na CBA.

No contexto de pessoas surdas, que tem como primeira língua a de sinais, Bueno et al. (2007) afirmam que o uso de AVAs pode ser apropriado para apoiar os processos de aprendizagem. No entanto, esse potencial só pode ser explorado se os cursos estiverem devidamente adaptados. Canal e García (2014) investigaram a acessibilidade de alguns tipos de questões suportadas pelo ambiente Moodle no contexto de estudantes surdos. Os resultados dessa investigação indicaram problemas no uso de vídeos, imagens, textos e personalização pelo usuário que vão além das diretrizes genéricas de acessibilidade na Web (e.g., Web Content Accessibility Guidelines [W3C 2014]).

No Brasil, estudantes surdos relatam dificuldades ao realizar o Exame Nacional do Ensino Médio (ENEM), que é realizado somente por meio de textos. Especialistas pedagogos afirmam que uma maneira de reverter essa situação é o estudante surdo ter a possibilidade de efetuar a prova com apoio de vídeos em Libras, com a prova feita em computador e com a presença de intérpretes para auxiliar em caso de dúvidas ${ }^{1}$.

Este trabalho propõe um conjunto de recomendações de acessibilidade para surdos dos tipos de questões usadas na CBA em AVAs. Este conjunto de recomendações articula trabalhos da literatura relacionados com o tema desta pesquisa. Algumas das recomendações foram mantidas na íntegra, enquanto outras foram adaptadas e reescritas no contexto dos tipos de questões usadas em AVAs para surdos.

Este artigo está organizado da seguinte maneira: a Seção 2 apresenta a revisão bibliográfica, a Seção 3 descreve o método utilizado neste trabalho; a Seção 4 sumariza os resultados; a Seção 5 apresenta uma discussão do trabalho realizado e, por fim, a Seção 6 apresenta as conclusões.

\section{Acessibilidade em CBA}

Thompson et al. (2002) analisaram as implicações do uso de CBA para alunos com deficiência e concluíram que o uso desse tipo de avaliação pode ter o potencial de melhorar a avaliação dos alunos, devido à possibilidade de incorporar certas acomodações, como por exemplo o uso de leitor de telas, que é uma maneira de realizar a leitura de informações na tela de maneira consistente para os estudantes com deficiência visual. No entanto, o uso de CBA pelos estudantes com deficiência pode ser desvantajoso se suas necessidades de acesso não forem consideradas desde o início do desenvolvimento de todo o processo.

No contexto dos surdos, Cawthon et al. (2011) realizaram um estudo em salas de aula com estudantes surdos onde o conteúdo das avaliações eram transmitidos aos estudantes pelo uso do computador por meio de vídeos em língua de sinais e as questões eram respondidas pelos estudantes de maneira escrita em papel. Resultados desse estudo mostram que os estudantes surdos obtiveram bom desempenho nos testes devido à compreensão do conteúdo transmitido nas avaliações pelos vídeos em língua de sinais.

\footnotetext{
${ }^{1}$ Uol.http://educacao.uol.com.br/noticias/2014/11/03/estudantes-surdos-tem-dificuldadepara-se-preparar-para-o-enem.htm
} 
No entanto, os trabalhos não abordam o uso dos tipos de questões em AVAs no contexto dos estudantes surdos.

Alguns trabalhos encontrados na literatura (e.g., Thompson et al. (2002); Beech et al. (2011)) indicam algumas recomendações para garantir que CBA possa ser utilizado por todos os estudantes, incluindo aqueles com deficiência. No entanto, esses trabalhos relatam apenas a acessibilidade em CBA de uma maneira genérica sem tratar algum tipo de deficiência de maneira específica e esses trabalhos estão relacionados ao WCAG. Dessa maneira, reforçam a abordagem de recomendações de acessibilidade de maneira mais genérica e abrangente, geralmente condizente com os princípios do Design Universal. Por outro lado, o WCAG, também proposto sob a visão do Design Universal, foca em websites em geral e, portanto, não tem o objetivo de esgotar todas as particularidades existentes nos diversos domínios do conhecimento. Portanto, uma abordagem de pesquisa comum é a extensão das diretrizes genéricas do WCAG para atender a domínios específicos (e.g., Hubert (2006)), tal como ocorre com as extensões às heurísticas genéricas de usabilidade como em Pinelle et al. (2009).

\section{Método}

Esta seção apresenta os passos metodológicos para (a) gerar o conjunto de recomendações de acessibilidade deste trabalho, (b) para a avaliação deste conjunto e (c) a geração do conjunto final de recomendações.

\subsection{Geração do conjunto de recomendações}

Para a geração do conjunto de recomendações que fazem parte deste trabalho foram realizados os seguintes passos metodológicos: seleção de conjuntos de recomendações, extração das recomendações, explicitação das recomendações, normalização das recomendações e, por último, geração do conjunto de recomendações.

Seleção de recomendações existentes. Baseada na seleção de trabalhos da literatura, divididos em três categorias: Recomendações de AVAs para surdos, Recomendações de acessibilidade em projetos de TICs para a alfabetização de surdos e Recomendações de acessibilidade em CBA. A primeira categoria se relaciona com o tema desta pesquisa devido ao fato de estar relacionada com a acessibilidade dos surdos na interação com AVAs (Bueno et al. (2007) Debevc et al. (2007); Debevc et al. (2012); Drigas et al. (2005); Khwaldeh et al. (2007); Pivetta et al. (2013); Pivetta et al. (2014); Straetz et al. (2004)). A segunda categoria envolve a construção de TICs, que podem dar suporte aos tipos de questões usadas na avaliação, que está inserida no contexto educacional (Abreu et al. (2010)). Já a terceira categoria envolve a acessibilidade na CBA, em que estão inseridos os tipos de questões (Beech (2011) Dolan et al. (2010); Russell et al. (2009); Russell (2011); Thompson et al. (2002)).

Extração e explicitação de recomendações. Alguns dos trabalhos selecionados apresentam recomendações de acessibilidade de maneira explícita nos textos, enquanto outros apresentam recomendações de maneira implícita. Nos trabalhos que apresentam as recomendações de maneira explícita, essas recomendações eram extraídas diretamente. Foram consideradas recomendações explícitas aquelas em que são apresentadas diretamente pelos trabalhos como recomendações de acessibilidade. Já nos trabalhos que possuem as recomendações de maneira implícita, foi realizada a extração 
dos trechos de textos que possuem evidências de orientações de acessibilidade. Após a extração dos trechos de textos, estes eram reescritos de maneira a promover a explicitação das recomendações de acessibilidade.

Normalização. Na etapa de normalização, o objetivo é dividir as recomendações que abordem mais de um tema em seu texto. A divisão das recomendações foi realizada por meio de uma quebra de recomendações. Por exemplo, se em uma recomendação existem duas orientações de acessibilidade, no processo de normalização há uma quebra nessa recomendação e, dessa maneira, passa a serem duas recomendações de acessibilidade. Ao identificar que algumas recomendações se repetem em diferentes trabalhos, foi realizada a junção das recomendações para que elas aparecessem somente uma vez no conjunto, com a respectiva identificação dos trabalhos que as propuseram.

Categorização. Nesta etapa, primeiramente foram criadas 9 categorias: 'Vídeo em Língua de Sinais', 'Apresentação Alternativa para Áudio, 'Apresentação Alternativa para Texto Escrito', 'Apresentação Alternativa para Conteúdo Visual', Conteúdo, Navegação, Dicionários e 'Glossários de Termos', 'Colaboração entre os Estudantes' e 'Feedback'. Essas categorias foram criadas de acordo com o tema com que cada recomendação está relacionada de maneira a agrupá-las.

Adaptação de recomendações. Algumas recomendações extraídas da literatura 'foram adaptadas para o contexto dos surdos. Por exemplo, a recomendação "3.Fornecer mecanismo para controle da velocidade da sinalização em língua de sinais" foi adaptada de Thompson et al. (2002), cuja recomendação original é: Capacidade de alterar a velocidade do áudio. A adaptação foi realizada devido ao fato de que o controle da velocidade do áudio não faz sentido ao usuário surdo, mas sim a possibilidade de alterar a velocidade da sinalização em língua de sinais.

Classificação de recomendações. Envolveu a classificação das recomendações de acordo com sua aplicação em tipos de questão e em perguntas e respostas. A classificação foi realizada devido a diferença na estrutura da criação da pergunta e da maneira de resposta nos tipos de questões. Por exemplo, a questão de múltipla Escolha é composta pelo seu enunciado e pelas alternativas de escolha, em que o professor é o responsável pela elaboração do enunciado e das alternativas. Já a questão do tipo lacuna, é composta pelo enunciado e pelos campos em branco ao longo da pergunta, onde deve ser preenchida a resposta. No tipo de questão lacuna, o professor é o responsável pela elaboração do enunciado e o estudante é quem deve preencher os espaços de resposta. Para isso, considerou-se como pergunta o que o professor é responsável por elaborar e como resposta o que o aluno é responsável em responder.

Criação de perfis e de sugestões de uso. Nesta etapa sugestões de uso das recomendações foram adicionadas conforme alguns perfis de usuários que foram nomeados de professor (a), estudante e desenvolvedor (a). A adição de sugestões de uso das recomendações por perfis de usuários tem o objetivo de clarificar e auxiliar a aplicação das recomendações de acessibilidade.

Avaliação do conjunto de recomendações. Foram realizadas duas etapas: a avaliação das recomendações por especialistas em TI e entrevista com pessoas surdas (em ambas assinaram um TCLE). A avaliação por especialistas envolveu 3 doutorandos e 3 mestrandos em Ciência da Computação. Dos 6 especialistas, 3 deles são 
pesquisadores de acessibilidade para surdos e 4 atuam na área de Interação HumanoComputador. Os especialistas foram convidados a formar duplas e avaliar a acessibilidade dos tipos de questões suportadas pelo Moodle 2.8, com o uso do conjunto de recomendações. Os especialistas anotaram em um formulário os problemas de acessibilidade encontrados bem como observações sobre o uso das recomendações. Por fim, os especialistas responderam um questionário sobre o uso das recomendações.

A entrevista foi realizada com duas professoras surdas que possuem mestrado e doutorado em educação e são especialistas na educação de surdos. Ambas atuam como docentes na Universidade Tecnológica Federal do Paraná e a entrevista contou com a presença de um intérprete. $\mathrm{O}$ pesquisador não apresentou as recomendações às professoras com o intuito de não induzir as respostas da entrevista. Ao mostrar e criar um exemplo de cada tipo de questão no Moodle 2.8, o pesquisador questionava as professoras sobre o que elas consideram que poderia ser alterado/adicionado para alcançar a acessibilidade no contexto dos surdos. A partir dos comentários das professoras entrevistadas, o pesquisador fazia perguntas referentes às observações apresentadas pelas professoras, com o objetivo de identificar se as recomendações eram, de alguma maneira, abordadas pelas professoras. A análise dos dados da entrevista envolveu o formulário de anotações do pesquisador e o áudio gravado da entrevista.

\section{Resultados}

Esta seção está organizada da seguinte maneira: resultados do processo de construção do conjunto de recomendações, da avaliação das recomendações por especialistas em TI e por pessoas surdas e do conjunto final de recomendações deste trabalho.

\subsection{Conjunto de recomendações}

O conjunto inicial de recomendações foi composto por 36 recomendações (a tabela 1 apresenta um exemplo de recomendação). Esse conjunto foi avaliado posteriormente.

Conforme a tabela 1, cada recomendação é identificada pela categoria a qual está relacionada, juntamente com o trabalho que a propôs. Cada recomendação também é identificada pela sua aplicação em perguntas e respostas referentes ao tipo de questão aos quais se aplica. As recomendações também apresentam sugestões de uso referentes aos 3 tipos de perfis criados.

\subsection{Avaliação do conjunto de recomendações}

A avaliação das recomendações foi realizada por especialistas em TI e por professoras surdas. Com o uso das recomendações, os especialistas encontraram problemas de acessibilidade nos tipos de questões avaliadas no Moodle. Os problemas encontrados por Canal e García (2014) foram novamente identificados pelos especialistas. Além dos problemas já reportados, os especialistas identificaram outros problemas de acessibilidade como, por exemplo, a ausência de dicionários e glossários de termos e de corretores ortográficos para elaboração dos tipos de questões e na resposta das questões que possuem campo de preenchimento, como a questão do tipo dissertação.

Sobre o questionário respondido pelos especialistas todos concordaram que o conjunto de recomendações os ajudou a identificar problemas de acessibilidade. Em relação ao conteúdo das recomendações, 4 afirmaram tê-las compreendido parcialmente. 
Os outros 2 afirmaram terem entendido plenamente o conteúdo das recomendações. Os 4 especialistas que entenderam parcialmente o conteúdo das recomendações, atribuíram isso ao fato de não terem conhecimento de alguns termos ou não sabiam diferenciar o seu significado, como por exemplo os termos hotspot e closed caption. Em relação à quantidade das recomendações do conjunto, todos os participantes consideraram que são muitas. No entanto, 5 especialistas consideraram essa quantidade necessária e, somente um considerou que há recomendações demais.

Tabela 1. Exemplo de recomendação

\begin{tabular}{|c|c|}
\hline Categoria & Vídeo em Língua de Sinais \\
\hline Recomendação & $\begin{array}{l}\text { 1. A qualidade do vídeo deve permitir que sejam captados } \\
\text { detalhes sobre os movimentos das mãos, olhos e boca. }\end{array}$ \\
\hline Fonte(s): & Debevc et al. (2007) \\
\hline $\begin{array}{l}\text { Aplicação: pergunta por tipo de } \\
\text { questão }\end{array}$ & $\begin{array}{l}\text { Associação, dissertação, lacuna, múltipla escolha, resposta curta } \\
\text { e verdadeiro/falso }\end{array}$ \\
\hline Aplicação: resposta por tipo de questão & Dissertação, lacuna e resposta curta \\
\hline $\begin{array}{l}\text { Sugestões de uso para os tipos de } \\
\text { questões em AVAs para surdos }\end{array}$ & $\begin{array}{l}\text { Professor (a): Possibilidade de o professor gravar a pergunta da } \\
\text { questão em língua de sinais, de maneira que sejam captados pelo } \\
\text { vídeo o movimento das mãos, olhos e boca; } \\
\text { Estudante: Possibilidade de o estudante gravar as respostas em } \\
\text { vídeo em língua de sinais, para alguns tipos de questões, como: } \\
\text { dissertação, lacuna e resposta curta; } \\
\text { Desenvolvedor (a): O desenvolvedor deve disponibilizar } \\
\text { ferramenta para gravação de vídeo das perguntas e das respostas. } \\
\text { O vídeo deve ter a dimensão necessária para capturar os } \\
\text { movimentos das mãos, olhos e boca. }\end{array}$ \\
\hline
\end{tabular}

Nenhum dos participantes identificou problemas de acessibilidade que já não fossem abordados pelas recomendações. Esta informação provê fortes indícios sobre a completude do conjunto de recomendações gerado neste trabalho. Quando perguntados se o conjunto de recomendações os havia ajudado a encontrar problemas de acessibilidade que não teriam identificado sem o conjunto, 2 dos especialistas concordaram plenamente, enquanto que os demais concordaram parcialmente. Por fim, 5 dos 6 especialistas concordaram plenamente que o conjunto de recomendações pode ser útil mesmo para profissionais de TI sem conhecimento prévio sobre acessibilidade para surdos. O outro especialista concordou parcialmente com esta afirmação.

Durante a realização da entrevista com as professoras surdas, as primeiras observações feitas por elas foram que os surdos possuem diferenças entre si. Enquanto alguns estudantes surdos possuem domínio do texto, outros possuem dificuldade e preferem interagir através da língua de sinais. Dessa maneira, as professoras acreditam que uma maneira de alcançar a acessibilidade nos tipos de questões é a possibilidade de se criar questões em texto e também com o uso de vídeos em língua de sinais e, assim, englobar as diferenças que possam existir entre os surdos. Esses comentários realizados pelas professoras são contemplados pelas recomendações que fazem parte das categorias 
"Vídeo em língua de sinais", "Apresentação alternativa para texto escrito" e “Apresentação alternativa para conteúdo visual”.

As professoras mencionaram uma situação em que estudantes surdos que frequentaram um curso em um AVA precisavam da ajuda de outros colegas que dominavam o português para traduzir o conteúdo para a língua de sinais. Ainda segundo as professoras, em alguns casos, os alunos surdos contratavam intérpretes para traduzir o conteúdo para língua de sinais e assim conseguir realizar as atividades do curso. Esta observação feita pelas professoras é contemplada pelas recomendações de acessibilidade que fazem parte das categorias, "Apresentação alternativa para texto escrito", "Vídeo em língua de sinais" e "Dicionários e glossários de termos". As professoras também afirmaram que o uso de dicionários traz benefícios. Recomendações sobre o uso de dicionários estão agrupadas na categoria "Dicionários e glossários de termos".

Quando perguntadas sobre a gravação do conteúdo da questão pelos docentes em língua de sinais diretamente no AVA, as professoras afirmaram que utilizariam esse recurso (abordado pelas recomendações da categoria "Vídeo em língua de sinais"). Por outro lado, as professoras afirmaram que a gravação de vídeos em língua de sinais pelos estudantes, diretamente no AVA, para responder as questões que possuem campo de preenchimento, poderia constranger alguns estudantes, por receio de cometerem erros. No entanto, elas acreditam que esse tipo de recurso promove a acessibilidade.

Em relação ao uso de imagens as professoras deram um exemplo de clicar na palavra e aparecer uma imagem e uma breve descrição para auxiliar na compreensão do significado da palavra (contemplada pela recomendação "19. Se um conceito pode ser descrito por meio de uma imagem, use a imagem e a sua descrição"). As professoras também afirmaram que a adição de imagens ao texto pode auxiliar na compreensão da questão pelos estudantes surdos (atendidas pela recomendação "9. Prover recursos visuais aos conteúdos apresentados em texto").

$\mathrm{Na}$ entrevista com as professoras não foi possível obter informações que comprovem a precisão de todas as 36 recomendações geradas neste trabalho, devido ao tempo de realização da entrevista (cerca de 1 hora). Entretanto, por meio dos dados coletados é possível identificar informações relevantes e relacionadas com as recomendações do conjunto inicial.

\subsection{Conjunto final de recomendações}

A partir da análise dos dados das atividades com especialistas e com as professoras, foi possível identificar informações sobre o uso das recomendações deste trabalho. De acordo com os resultados da avaliação realizada pelos especialistas em TI, identificou-se a sugestão de exclusão de algumas recomendações (e.g., "17. Textos devem ser escritos com frases tão simples quanto possível", "32. Uso de configuração [física] individual se o método de resposta distrai os outros estudantes"). De acordo com os especialistas estas recomendações ficam a cargo do docente e não da ferramenta. Portanto, 34 recomendações compõem o conjunto final e podem ser acessadas em bit.ly/1HWb8Gh.

\section{Discussão}

A avaliação realizada pelos especialistas em TI com o uso das recomendações, bem como a entrevista realizada com as professoras surdas, possibilitaram a obtenção de 
informações relevantes sobre a precisão, a clareza e a completude do conjunto de recomendações, proposto neste trabalho. Os resultados obtidos da avaliação pelos especialistas indicam que, mesmo AVAs amplamente adotados e com comunidades de desenvolvedores ativas, como o Moodle, ainda possuem diversos problemas de acessibilidade relacionados ao uso dos tipos de questões. A inclusão de estudantes surdos passa pela solução dos problemas de acessibilidade aqui identificados.

Os problemas de acessibilidade encontrados na avaliação pelos especialistas tem relação com os problemas identificados por Canal e García (2014). Por exemplo, (a) a impossibilidade de adição de outras mídias, que não texto, às alternativas de resposta disponíveis ao estudante no tipo de questão associação e (b) a impossibilidade de adição de legendas em vídeos em todos os tipos de questões suportadas pelo Moodle. Entretanto, devido ao fato de o conjunto de recomendações ser composto por um número maior de recomendações do que as recomendações utilizadas no trabalho de Canal e García (2014), os especialistas em TI encontraram um número maior de problemas e, também, identificaram problemas adicionais às recomendações utilizadas por Canal e García (2014). Portanto, pode-se concluir que o conjunto de recomendações possibilita a identificação de uma variedade maior de problemas de acessibilidade nos tipos de questões usadas em AVAs.

Com relação ao uso das recomendações no processo de avaliação realizada pelos especialistas, os resultados apontam pontos positivos com relação à precisão das recomendações. Essa afirmação se sustenta pela coerência dos problemas apontados pelos especialistas com o estudo preliminar realizado por Canal e García (2014). Os especialistas também confirmaram que compreenderam o conteúdo das recomendações e a maioria dos especialistas afirmaram que apesar do conjunto de recomendações ser extenso, as recomendações que compõem esse conjunto são necessárias. Portanto, esses resultados indicam que o uso das recomendações foi recebido de uma maneira positiva pelos especialistas e que o conjunto de recomendações é preciso, claro e amplo.

De acordo com a entrevista realizada com as professoras é possível afirmar que o conjunto de recomendações é amplo, uma vez que as professoras não abordaram qualquer outra informação que não seja coberta pelas recomendações. É possível afirmar que o conjunto de recomendações é preciso, uma vez que as orientações feitas pelas professoras são coerentes com aquelas presentes nas recomendações. $\mathrm{O}$ aspecto de clareza não foi avaliado na entrevista com as professoras devido ao fato de que elas não tiveram acesso ao conjunto de recomendações.

Em relação ao público alvo, o conjunto de recomendações atende à diversidade do público surdo, pois está focado em prover uma gama de recursos configuráveis, selecionados e omitidos pelos estudantes de acordo com suas necessidades e preferências. Esta abordagem é semelhante às empregadas pelos conjuntos de diretrizes para acessibilidade web, como o WCAG, o Section $508^{2}$ e o E-Mag ${ }^{3}$. O conjunto de recomendações deste trabalho pode ser considerado mais completo do que os conjuntos de recomendações originais uma vez que problemas de acessibilidade encontrados envolvem recomendações propostas por trabalhos diferentes.

\footnotetext{
${ }^{2}$ http://www.section508.gov/

${ }^{3}$ http://www.governoeletronico.gov.br/acoes-e-projetos/e-MAG
} 


\section{Conclusões}

Este trabalho investigou a acessibilidade dos tipos de questões usadas em CBA em AVAs no contexto dos estudantes surdos. Como produto deste estudo, foi proposto um conjunto de recomendações que possibilitam a melhoria da acessibilidade nos tipos de questões para estudantes surdos, consequentemente propiciando condições para a promoção da inclusão dos estudantes surdos. Além disso, as recomendações geradas neste trabalho levam em consideração as diferenças entre os tipos de estudantes surdos ao propor o provimento de recursos personalizados e alternativos.

Por meio da avaliação pelos especialistas em TI e da entrevista realizada com a participação de professoras que são surdas é possível concluir de maneira positiva sobre a clareza, a precisão e a completude do conjunto de recomendações que fazem parte deste trabalho. Sendo assim, há indícios para afirmar que este trabalho tem o potencial de prover instrumentos de suporte à construção de recursos CBA acessíveis e, por consequência, de uma sociedade mais inclusiva.

\section{Referências}

Abreu, P. M.; Prates, R. e O. Bernardino, E. L. A. (2010). "Recomendações de acessibilidade para projetos de TICs para alfabetização de crianças surdas". Anais do XXXVII Seminário Integrado de Software e Hardware. CSBC, 1-7.

Beech, M. (2011). "Computer-based testing accommodations for students with disabilities". Technical report. Ph.D. Learning Systems Institute. Florida State University. Bureau of Exceptional Education and Student Services Florida Department of Education.

Bueno, J. F., Castilho, D, F, R, J., Garcia, S. e Borrego, R. (2007). "E-learning content adaptation for deaf students". In Proc. $12^{\text {th }}$ annual SIGCSE conference on Innovation and technology in computer science education. ACM, 271-275.

Canal, M.C. e García, L.S. (2014). "Research on Accessibility of Question Modalities Used in Computer-Based Assessment (CBA) for Deaf Education". Universal Access in Human-Computer Interaction. Universal Access to Information and Knowledge. Lecture Notes in Computer Science, Volume 8514, pp 265-276.

Cawthon, S.W., Winton S.M., Garberoglio, C.L. e Gobble, M.E. (2011). "The effects of american sign language as an assessment accommodation for students who are deaf or hard of hearing". Journal of Deaf Studies and Deaf Education, 16(2):198-211.

Debevc, M., Povalej, M., Verli£, M. e Stjepanovi£, Z. (2007). "Exploring usability and accessibility of an e-learning system for improving computer literacy". New Trends in ICT \& Accessibility, 119-124.

Debevc, M., Stjepanovi£, Z. e Holzinger, A. (2012). "Development and evaluation of an e-learning course for deaf and hard of hearing based on the advanced adapted pedagogical index method". Interactive Learning Environments, 22(1):35-50.

Dolan, R.P., Burling, K.S., Harms, M., Beck, R., Hanna, E., Jude, J., Murray, E.A., Rose, D.H. e Way, W. (2010). "Universal design for computer-based testing guidelines". Technical report. Pearson and CAST. 
Drigas, A.S., Kouremenos, D., Kouremenos, S. e Vrettaros, J. (2005). "An e-learning system for the deaf people. Information Technology Based Higher Education and Training". ITHET 2005. $6^{\text {th }}$ International Conference on, T2C/17-T2C/21.

Hubert, R. (2006). "Accessibility and usability guidelines for mobile devices in home health monitoring". SIGACCESS Access. Comput., (84):26-29.

Khwaldeh S., Matar, N. e IHunaith, Z. (2007). "Interactivity in deaf classroom using centralised elearning system in jordan”. PGNet.

Luephattanasuk, N., Suchato, A. e Punyabukkana, P. (2011). "Accessible QTI presentation for web-based elearning". In Proceedings of the International CrossDisciplinary Conference on Web Accessibility. ACM, Article 26.

Pinelle, D., Wong, N., Stach, T. e Gutwin, C. (2009). "Usability heuristics for networked multiplayer games". Proceedings of the ACM 2009 International Conference on Supporting Group Work, ACM, 169-178 .

Pivetta, E.M., Saito, D.S., Almeida, A.M.P. e Ulbricht, V.R. (2013). “Contribuições para o design de interface de um ambiente virtual de ensino aprendizagem acessível a surdos". Infodesign, 10:193-206.

Pivetta, E.M., Saito, D.S. e Ulbricht, V.R. (2014). "Surdos e acessibilidade: análise de um ambiente virtual de ensino e aprendizagem". Revista Brasileira de Educação Especial, 20:147-162.

Russell, M. (2011). "Digital test delivery: Empowering accessible test design to increase test validity for all students". White paper, Bill \& Melinda Gates Foundation.

Russell, M., Hoffmann, T. e Higgins, J. (2009). "Meeting the needs of all students: A universal design approach to computer-based testing". Journal of Online Education, $5(4)$.

Russell, M., Kavanaugh, M., Masters, J., Higgins, J. e Hoffmann, T. (2009). "Computer-based signing accommodations: Comparing a recorded human with an avatar.” Journal of Applied Testing Technology, 10(3).

Sim, G., Holifield, P. e Brown, M. (2004). "Implementation of computer assisted assessment: Lessons from the literature". Association for Learning Technology Journal, 12(3), 215-229.

Straetz, K., Kaibel, A., Raithel, V., Specht, M., Grote, K. e Kramer, F. (2004). "An elearning environment for deaf adults". Conference Proc. $8^{\text {th }}$ ERCIM Workshop User Interfaces for All.

Thompson, S.J., Thurlow, M.L., Quenemoen, R.F. e Lehr, C.A. (2002). “Access to computer-based testing for students with disabilities". (synthesis report 45) Technical report. University of Minnesota, National Center on Educational Outcomes, Minneapolis.

W3C. (2014) "Web content accessibility guidelines (WCAG) 2.0." http://www.w3.org/TR/WCAG20/. 